\title{
Identification of the Control Chart Patterns Using the Optimized Adaptive Neuro-Fuzzy Inference System
}

\author{
Abdolhakim Nikpey, Somayeh Mirzaei \\ Shams University, Gonbad Kavous, Iran \\ Email: Nikpey72@gmail.com, somayeh.mirzaei.shams@gmail.com \\ Masoud Pourmandi \\ Ferdowsi University, Mashhad, Iran \\ Email: massoud.pourmandi@gmail.com
}

Jalil Addeh

Payam Noor University, Gonbad Kavous, Iran

Email: Jalil_addeh@yahoo.com

\begin{abstract}
Unnatural patterns in the control charts can be associated with a specific set of assignable causes for process variation. Hence pattern recognition is very useful in identifying process problem. This paper presents a novel hybrid intelligent method for recognition of common types of control chart patterns (CCPs). The proposed method includes three main modules: the feature extraction module, the classifier module and the optimization module. In the feature extraction module, a proper set of the shape features and statistical features is proposed as the efficient characteristic of the patterns. In the classifier module adaptive neuro-fuzzy inference system (ANFIS) is investigated. In ANFIS training, the vector of radius has very important role for its recognition accuracy. Therefore, in the optimization module, cuckoo optimization algorithm (COA) is proposed for finding of optimum vector of radius. Simulation results show that the proposed system has high recognition accuracy.
\end{abstract}

Index Terms - ANFIS, Control chart patterns, Shape features, Statistical feature, COA.

\section{INTRODUCTION}

Control chart has been widely used in modern industrial and service organization. In recent years, various kinds of control charts have been developed according to different quality attributes and control targets. Monitoring process fluctuation with control charts is first proposed by Shewhart in 1924. It is believed that the process fluctuation involves abnormal changes due to assign able causes and normal changes due to non- assignable causes. Therefore, automatically recognizing control chart patterns (CCPs) is an essential issue for identifying the process fluctuation effectively. CCPs can exhibit six common types of pattern: normal (NOR), cyclic (CYC), increasing trend (IT), decreasing trend (DT), upward shift (US), and downward shift (DS). Except for normal patterns, all other patterns indicate that the process being monitored is not functioning correctly and requires adjustment. Fig. 1 shows these six types of patterns [1].
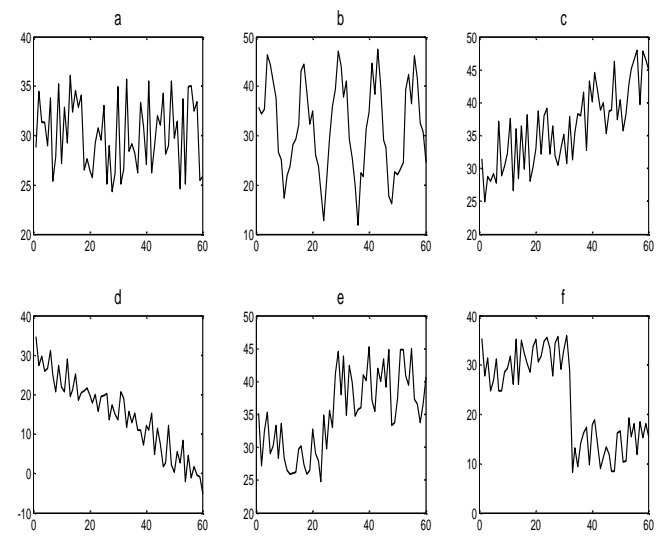

Fig. 1. Six various basic patterns of control charts: (a) Normal pattern (b) Cyclic pattern, (c) Upward trend, and (d) Downward Trend, (e) Upward shift, (f) Downward shift

In recent years, several studies have been performed for recognition of the unnatural patterns. Some of the researchers used the expert systems [2,3]. The advantage of an expert system or rule-based system is that it contains the information explicitly. If required, the rules can be modified and updated easily. However, the use of rules based on statistical properties has the difficulty that similar statistical properties may be derived for some patterns of different classes, which may create problems of incorrect recognition. Also, ANNs have been widely applied for classifiers. ANNs can be simply categorized into two groups comprising supervised and unsupervised. Most researchers [4- 6] have used supervised ANNs, such 
as multi layer perceptron (MLP), radial basis function (RBF), and learning vector quantization (LVQ), to classify different types of CCPs. Furthermore, unsupervised methods, e.g. self-organized maps (SOM) and adaptive resonance theory (ART) have been applied to fulfill the same objective in other studies [7, 8]. The advantage with neural network is that it is capable of handling noisy measurements requiring no assumption about the statistical distribution of the monitored data. It learns to recognize patterns directly through typical example patterns during a training phase. One disadvantage with neural network is the difficulty in understanding how a particular classification decision has been reached and also in determining the details of how a given pattern resembles with a particular class. In addition, there is no systematic way to select the topology and architecture of a neural network. In general, this has to be found empirically, which can be time consuming.

Most the existing techniques used the unprocessed data as the inputs of CCPs recognition system. The use of unprocessed CCP data has further many problems such as the amount of data to be processed is large. On the other hand, the approaches which use features are more flexible to deal with a complex process problem, especially when no prior information is available. If the features represent the characteristic of patterns explicitly and if their components are reproducible with the process conditions, the classifier recognition accuracy will increase [9]. Further, if the feature is amenable to reasoning, it will help in understanding how a particular decision was made and thus makes the recognition process a transparent process. Features could be obtained in various forms, including principal component analysis shape features $[10,11]$, multi-resolution wavelet analysis $[12,13]$ and statistical features [14]. Pham and Wani [10] introduced feature based control chart pattern recognition. Nine geometric features were proposed: slope, number of mean crossings, number of least-square line crossings, cyclic membership, average slope of the line segments, slope difference, and three different measures for area. The scheme was aimed at improving the performance of the pattern recognizer by presenting a smaller input vector (features). Gauri and Chakraborty [11] also present a set of seven most useful features that are selected from a large number of potentially useful features using a CART-based systematic approach. Based on these selected features, eight most commonly observed CCPs are recognized using heuristic and ANN techniques. Chen [12] presents a hybrid approach by integrating wavelet method and neural network for on-line recognition of concurrent CCPs. In the hybrid system, concurrent CCPs are first preprocessed by a wavelet transform to decompose the concurrent patterns into different levels or patterns, and then the corresponding features are fed into back-propagation ANN classifiers for pattern recognition. Hassan et al. [13] conducted an experimental study to use BPNs for identifying six types of basic SPC patterns, where the performances of two BPN recognizers using statistical features and raw data as input feature, respectively, were compared. The results indicated that the BPN using statistical features as input vectors has better performance than those of the other BPN using raw data as input vectors.

Based on the published papers, there exist some important issues in the design of automatic CCPs recognition system which if suitably addressed, lead to the development of more efficient recognizers. One of these issues is the extraction of the features. In this paper for obtaining the compact set of features which capture the prominent characteristics of the CCPs in a relatively small number of the components, the statistical and shape features are applied. These features are presented in Section 2.

Another issue is related to the choice of the classification approach to be adopted. The proposed method uses fuzzy rules for recognition task. In the proposed method, an expert system has been developed which has fuzzy rules obtained by adaptive neuro-fuzzy inference system (ANFIS). ANFIS represent the promising new generation of information processing systems. Adaptive network based fuzzy inference systems are good at tasks such as pattern matching and classification, function approximation, optimization and data clustering, while traditional computers, because of their architecture, are inefficient at these tasks, especially pattern-matching tasks [14-18].

The structure of the proposed classifier is shown in the Fig. 2. It can be seen that this system is composed of the two major decision layers. In this system, patterns are divided into three binary groups using Statistical feature and classifier 1 in the first layer. In the second layer the recognition process is done by shape features and classifiers in each group.

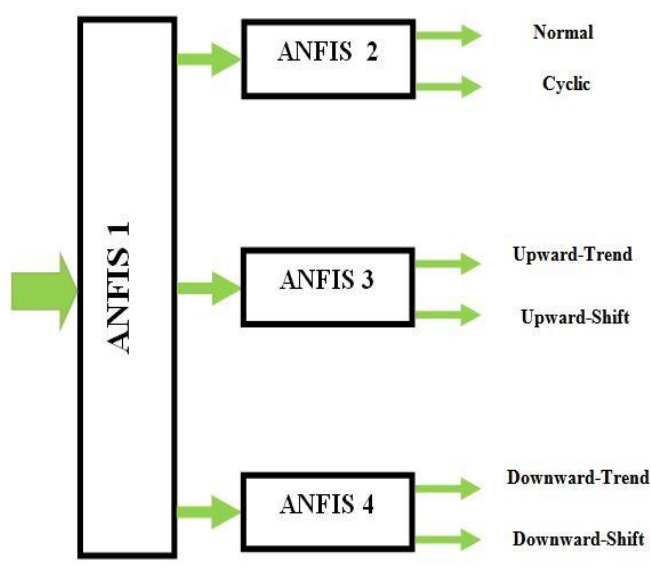

Fig.2. Structure of proposed Classifier

Also, proposed method shown in Fig 3. In ANFIS training process, the radius of clusters has high efficiency on the performance of system. For this respect, cuckoo optimization algorithm (COA) is chosen as an optimization technique to optimize the ANFIS parameter. This technique will improve the ANFIS performance.

The rest of paper is organized as follows. Section 2 explains the feature extraction. Section 3 presents the needed concepts including the ANFIS and cuckoo optimization algorithm. Section 4 describes the proposed 
method. Section 5, shows simulation results and finally Section 6 concludes the paper.

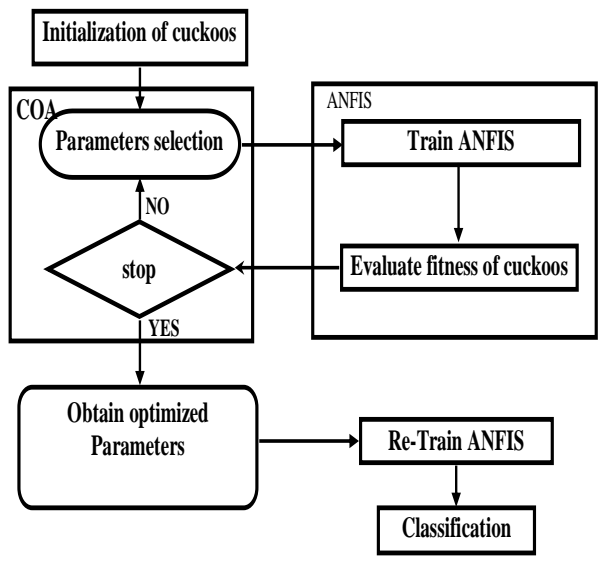

Fig. 3. Proposed method

\section{FEATURE EXTRACTION}

Features represent the format of the CCPs. As we know, different types of CCP have different properties; therefore finding the suitable features in order to identify them is a difficult task. In the signal recognition area, choosing the good features not only enables the classifier to distinguish more and higher CCPs, but also helps reduce the complexity of the classifier. In this paper, for the feature extraction module we have used a suitable set of features that consists of both shaping and statistical information of the CCPs. Figs. 4-10 show these features. These features are briefly described as follows.

\section{A. Statistical Feature}

Some statistical features are mean, standard deviation, skewness, kurtosis, and autocorrelation. In this paper we have used mean as feature1.

Feature1 (mean): Its mathematical form is shown below:

$$
\text { mean }=\frac{\sum_{i=1}^{n} X_{i}}{n}
$$

where $X_{i}$ represent the input (reference) vector and $n$ is the total length of the observing window.

\section{B. Shape features}

In [10], the authors have introduced nine shape features for discrimination of the CCPs. In this paper, three of these features are considered as features 2,3 and 4 . Feature 5 is proposed in this paper.

Feature2 (APML): the area between the pattern and the mean line. The APML is lowest for a normal pattern.
Thus, this feature differentiates between normal and other patterns.

Feature3 (APSL): the area between the pattern and its least-square line. Cyclic and shift patterns have a higher APSL value than normal and trend patterns and therefore the APSL can be used to differentiate cyclic and shift patterns from normal and trend patterns.

Feature4 (ASS): the area between the least-square line and the line segments. The value of this feature is approximately zero for a trend pattern and is higher for a shift pattern. This feature thus differentiates trend patterns from shift patterns.

Feature5 (MVSASTI): the maximum value of variation in signal amplitude in a short time interval (proposed shape feature). As it is depicted in Fig. 1, the maximum variation of signal amplitude in a time interval of 60 second is approximately identical in both shift and trend patterns. But in shift pattern, there is a large amount of variation in signal amplitude in a part of signal and in a short time interval which is not existed in the trend pattern. This difference could be used for separating these two patterns. So, the maximum of signal variation is calculated in a short time interval like $\mathrm{T}$. beginning from $\mathrm{t}=0$, the maximum value of signal variation is calculated, from $\mathrm{t}=0$ to $\mathrm{t}=\mathrm{t}+\mathrm{T}$. This process is proceeded for $\mathrm{t}=1, \ldots$, $60-T$. Finally the maximum variation in different time interval is selected as a desired feature. $\mathrm{T}$ could be any number between 5 to 10 . This shape feature is new in this area. In this study $\mathrm{t}=7 \mathrm{sec}$ is selected.

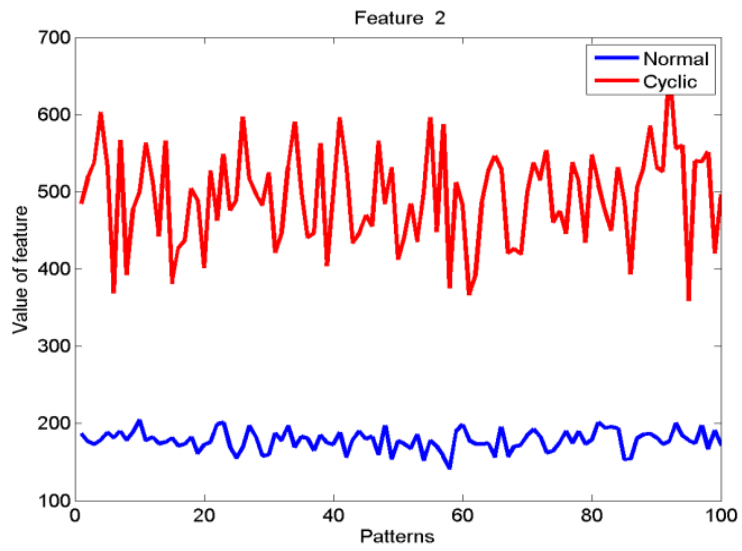

Fig. 4. APML of Normal and Cyclic Patterns

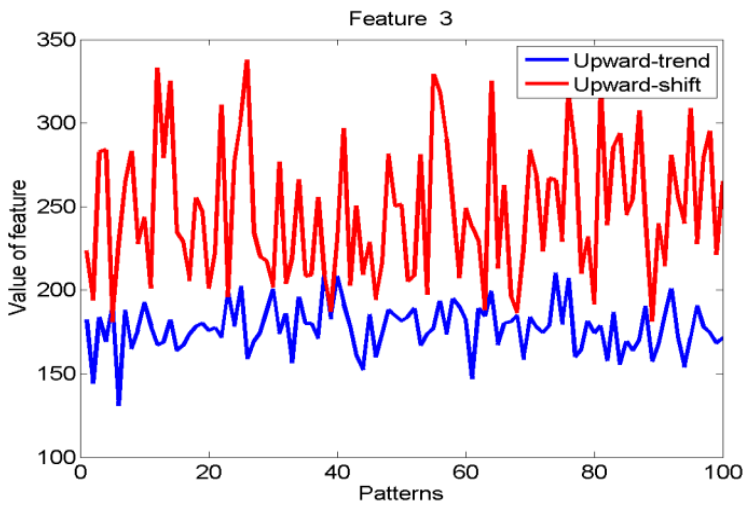

Fig 5. APSL of upward-trend and upward-shift patterns 


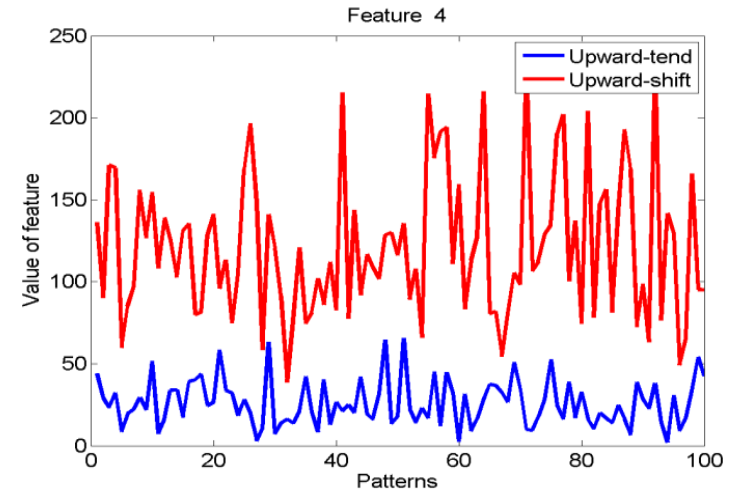

Fig 6. ASS of upward-trend and upward-shift patterns

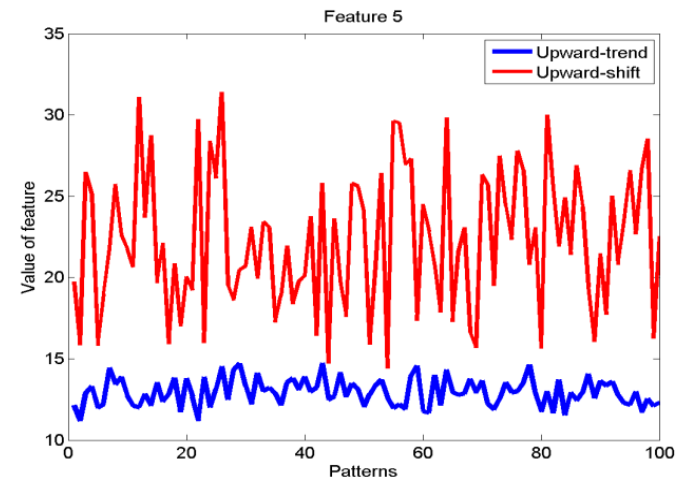

Fig 7. MVSASTI of upward-trend and upward-shift patterns

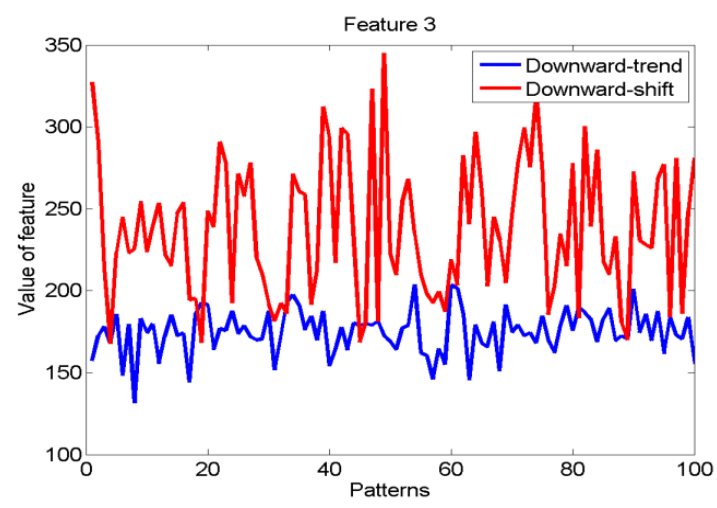

Fig 8. APSL of downward-trend and downward-shift patterns

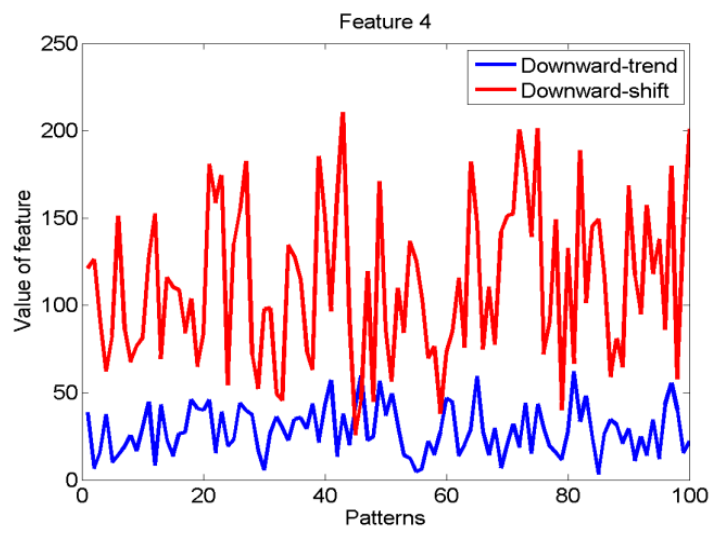

Fig 9. ASS of downward-trend and downward-shift patterns

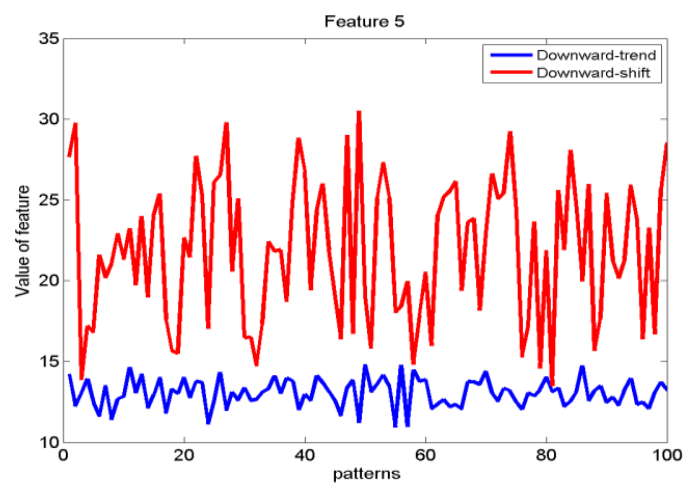

Fig 10. MVSASTI of downward-trend and downward-shift patterns

\section{NEEDED CONCEPTS}

\section{A. Adaptive network based fuzzy inference system (ANFIS)}

The adaptive network based fuzzy inference system (ANFIS) represents a useful neural network approach for the solution of function approximation problems. Data driven procedures for the synthesis of ANFIS networks are typically based on clustering a training set of numerical samples of the unknown function to be approximated. Since introduction, ANFIS networks have been successfully applied to classification tasks, rulebased process controls, pattern recognition problems and the like. Here a fuzzy inference system comprises of the fuzzy model [19, 20] proposed by Takagi, Sugeno and Kang to formalize a systematic approach to generate fuzzy rules from an input output data set. More details regarding ANFIS can be found in [21-29].

\section{B. Cuckoo optimization algorithm (COA)}

Fig. 11 shows a flowchart of the cuckoo optimization algorithm. Like other evolutionary algorithms, the COA starts with an initial population of cuckoos [30]. These initial cuckoos have some eggs to lay in some host birds' nests. Some of these eggs which are more similar to the host bird's eggs have the opportunity to grow up and become a mature cuckoo. Other eggs are detected by host birds and are killed. The grown eggs reveal the suitability of the nests in that area. The more eggs survive in an area, the more profit is gained in that area. So the position in which more eggs survive will be the term that COA is going to optimize.

Cuckoos search for the most suitable area to lay eggs in order to maximize their eggs survival rate. After remained eggs grow and turn into a mature cuckoo, they make some societies. Each society has its habitat region to live in. The best habitat of all societies will be the destination for the cuckoos in other societies. Then they immigrate toward this best habitat. They will inhabit somewhere near the best habitat. Considering the number of eggs each cuckoo has and also the cuckoo's distance to 
the goal point (best habitat), some egg laying radii is dedicated to it. Then, cuckoo starts to lay eggs in some random nests inside her egg laying radius. This process continues until the best position with maximum profit value is obtained and most of the cuckoo population is gathered around the same position. More details regarding COA can be found in [30].

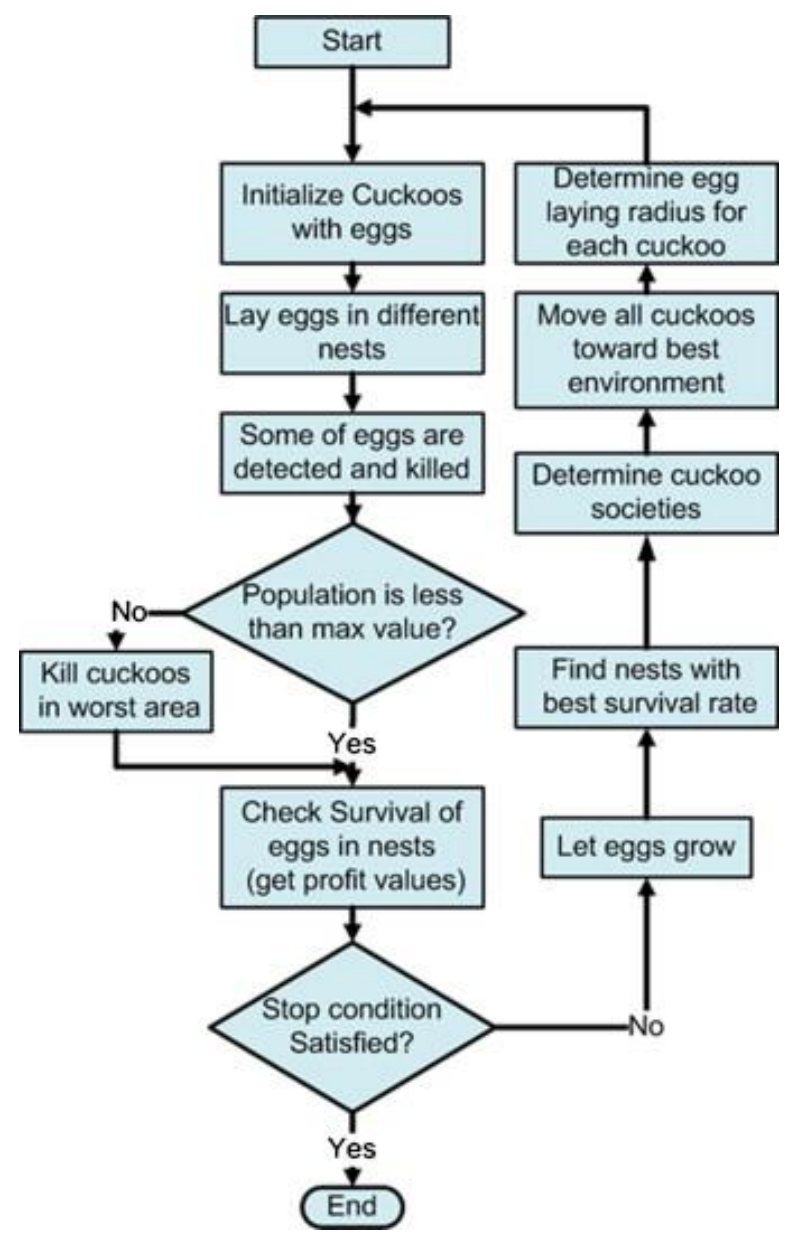

Fig. 11. Flowchart of Cuckoo Optimization Algorithm

\section{Proposed Method}

In this section, it is described the design of proposed method for CCPs. The structure of the proposed classifier is shown in the Fig. 2. It can be seen that this system is composed of the two major decision layers. In first layer sextuple patterns of control chart are divided to three groups of double patterns. This layer uses the statistical feature and divides the input patterns into three binary groups. As indicated in Fig. 12, the value of the used statistical feature of the member of the each group is close to each other. These binary groups are: normal and cyclic patterns (Group1, blue lines), upward shift and upward trend patterns (Group2, green lines), downward shift and downward trend patterns (Group3, red lines).

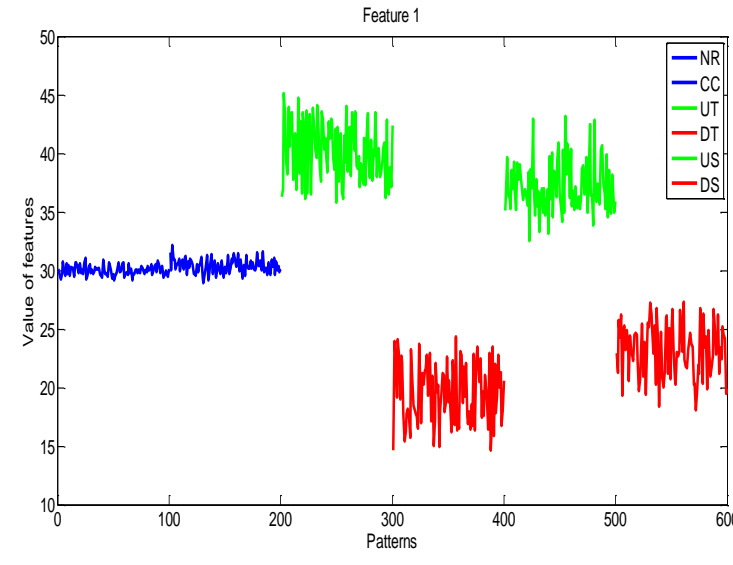

Fig.12. Mean of CCPs (Patterns from 1 to 100 are Normal patterns, Cyclic:101-200,Up-trend:201-300,Down-trend:301-400,Up-shift:401500, Down-shift:501-600).

In this layer because of using proper stochastic feature (mean), we are facing to a very easy classification problem. In the second layer, ANFIS2 is trained to separate normal and cyclic pattern. According to the Fig. 4 the separation of these patterns are very easy. Because the values of the feature 2 are completely different for the normal and the cyclic patterns. For separation of the members of the Group2, i.e. upward shift and upward trend patterns, the Feature3, the Feature 4 and the Feature 5 are used by the ANFIS3. ANFIS3 is trained to separate upward shift and upward trend patterns. For separation of the members of the Group3, the Feature3, the Feature 4 and the Feature 5 are used by the ANFIS4.

The proposed method is a COA based ANFIS classification technique. The structure of the proposed method is shown in the Fig. 3. This approach selects the optimum vector of radius.

The ANFIS model was developed using MATLAB Fuzzy Logic Toolbox (2009). A subtractive fuzzy clustering was generated to establish a rule base relationship between the input and output parameters. The data was divided into groups called as clusters using the subtractive clustering method to generate fuzzy inference system. In this study, the Sugeno-type fuzzy inference system was implemented to obtain a concise representation of a system's behavior with a minimum number of rules. The linear least square estimation was used to determine each rule's consequent equation. A radius value was given in the MATLAB program to specify the cluster center's range of influence to all data dimensions of both input and output. If the cluster radius was specified a small number, then there will be many small clusters in the data that results in many rules. In contrast, specifying a large cluster radius will yield a few large clusters in the data resulting in fewer rules [16]. For example, if the data dimension is 3 (e.g., input has two columns and output has one column), radii $=\left[\begin{array}{lll}0.5 & 0.4 & 0.3\end{array}\right]$ specifies that the ranges of influence in the first, second, and third data dimensions (i.e., the first column of input, 
the second column of input, and the column of output) are $0.5,0.4$, and 0.3 times the width of the data space, respectively. Fig. 13 shows the sample cuckoo.

$$
\text { Cuckoo }=\left[\text { radius }_{1}, \text { radius }_{2}, \ldots . ., \text { radius }_{p}\right]
$$

Fig. 13. Sample of particle

\section{Simulation Results}

In this section we evaluate the performance of proposed recognizer. For this purpose we have used the practical and real world data [31]. This dataset contains 600 examples of control charts.

For this study, we have used $60 \%$ of data for training the classifier and the rest for testing. The easiest way to assess the performance rate is to choose a test set independent of the training set and validation set to classify its examples, count the examples that have been correctly classified and divide by the size of the test set. The proportion of test-set examples that are classified correctly to the total samples, estimates the performance of recognizer for each pattern. In order to achieve the recognition accuracy (RA) of system, one needs to compute the average value of the performances of the CCPs.

\section{A. Performance of recognizer without optimization}

First we have evaluated the performance of the recognizer without optimization. Table 1 shows the recognition accuracy (RA) of non-optimized systems. As reported in Table 1, 97.43\% recognition accuracy is achieved.

Table 1. Recognition accuracy of the recognizer without optimization

\begin{tabular}{|c|c|c|c|}
\hline \multirow{2}{*}{ Classifier } & & RA (\%) & \\
\cline { 2 - 4 } & Min & Mean & Max \\
\hline ANFIS & 95.41 & 97.43 & 97.91 \\
\hline
\end{tabular}

In order to indicate the details of the recognition for each pattern, the confusion matrix of the recognizer is shown by Table 2. As we know, the values in the diagonal of confusion matrix show the correct performance of recognizer for each pattern. In other words, these value show that how many of considered pattern are recognized correctly by the system. The other values show the mistakes of system. For example, look at the third row of confusion matrix. The value of $96.5 \%$ shows the percentage of correct recognition of upward trend pattern and the value of $3.5 \%$ shows that this type of pattern is wrongly recognized with upward shift pattern. In order to achieve the recognition accuracy (RA) of system, it is needed to compute the average value of that appears in diagonal.

\section{B. Performance of recognizer with optimization}

Next, we apply COA to find the optimum parameters of the ANFIS. Table 3 shows the COA parameters. These values obtained after several experiments. Table 4 shows the RA of optimized system. From Table 1 and Table 4 it

Table 2. Confusion matrix for $97.43 \%$

\begin{tabular}{|c|c|c|c|c|c|c|}
\hline & NR & CC & UT & DT & US & DS \\
\hline NR & 100 & 0 & 0 & 0 & 0 & 0 \\
\hline CC & 0 & 100 & 0 & 0 & 0 & 0 \\
\hline UT & 0 & 0 & 96.5 & 0 & 3.5 & 0 \\
\hline DT & 0 & 0 & 0 & 96.0 & 0 & 4.0 \\
\hline US & 0 & 0 & 2.5 & 0 & 97.5 & 0 \\
\hline DS & 0 & 0 & 0 & 4.5 & 0 & 95.5 \\
\hline
\end{tabular}

can be found that RA is increased from $97.54 \%$ (in case of the non-optimized system) to $99.58 \%$. It can be seen that the optimization improves the performance of recognizer significantly. Also Table 5 shows the confusion matrix.

Table 3. Coefficient values in the COA

\begin{tabular}{|l|c|}
\hline Number of Cuckoos & 50 \\
\hline Minimum number of eggs & 2 \\
\hline Maximum number Of eggs & 5 \\
\hline Number of clusters & 2 \\
\hline Maximum number Of Cuckoos & 200 \\
\hline$\alpha$ & 40 \\
\hline$\lambda$ & 0.1 \\
\hline Maximum iteration & 100 \\
\hline
\end{tabular}

Table 4. Recognition accuracy of the recognizer with optimization

\begin{tabular}{|c|c|c|c|}
\hline \multirow{2}{*}{ Classifier } & & RA (\%) & \\
\cline { 2 - 4 } & Min & Mean & Max \\
\hline ANFIS & 99.58 & 99.58 & 99.58 \\
\hline
\end{tabular}

Table 5. Confusion matrix for $99.58 \%$

\begin{tabular}{|c|c|c|c|c|c|c|}
\hline & NR & CC & UT & DT & US & DS \\
\hline NR & 100 & 0 & 0 & 0 & 0 & 0 \\
\hline CC & 0 & 100 & 0 & 0 & 0 & 0 \\
\hline UT & 0 & 0 & 97.48 & 0 & 2.52 & 0 \\
\hline DT & 0 & 0 & 0 & 100 & 0 & 0 \\
\hline US & 0 & 0 & 0 & 0 & 100 & 0 \\
\hline DS & 0 & 0 & 0 & 0 & 0 & 100 \\
\hline
\end{tabular}

In order to compare the performance of COA with another nature inspired algorithms, we have used several nature inspired algorithms such as artificial bee colony (ABC) [32], genetic algorithm (GA) [33], particle swarm optimization (PSO) [34] and imperialist competitive algorithm (ICA) [35] to evolve the proposed method. According to results in Table 6, the best accuracy obtained for the test set by COA-ANFIS is $99.58 \%$.It can be seen that the success rates of COA is higher than the performance of other nature inspired algorithms.

Table 6. Comparison among the performance of different optimization algorithms

\begin{tabular}{|c|c|}
\hline Classifier & Recognition accuracy (\%) \\
\hline ABC-ANFIS & 99.24 \\
\hline GA-ANFIS & 98.45 \\
\hline PSO-ANFIS & 99.03 \\
\hline ICA-ANFIS & 99.17 \\
\hline COA-ANFIS & 99.58 \\
\hline
\end{tabular}




\section{Performance evaluation with optimization in different runs}

In this sub-section, for evaluating the performance of the COA, five different runs have been performed. Fig. 14 shows a typical increase of the fitness (classification accuracy) of the best individual fitness of the population obtained from proposed system for different runs. As indicated in this figure, its fitness curves gradually improved from iteration 0 to 100 , and exhibited no significant improvements after iteration 70 for the five different runs. The optimal stopping iteration to get the highest validation accuracy for the five different runs was around iteration 60-70.

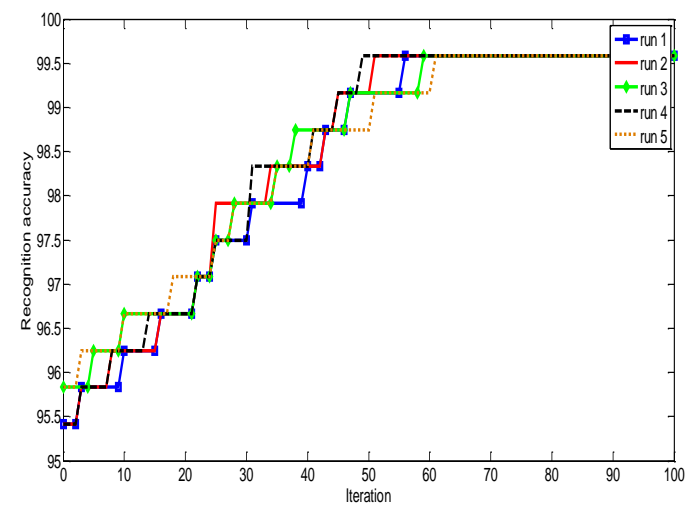

Fig 14.Evolution of fitness function for different runs.

\section{Effects of the COA parameters on the performance of the method}

In this subsection we have analyzed the sensitivity of the recognition system with respect to the $\alpha$ and $\lambda$, which control the behavior, and thus, the goodness of the COAsearch process. The achieved results for 10 set of parameters are shown in Table 7. For surveying the influence of parameters in each case, ANFIS is tested 50 times independently. The average of best obtained value is depicted in table. It illustrates that this hybrid system have a little dependency on variation of the parameters.

Table 7. Recognition accuracy of the recognizer for different values of parameters

\begin{tabular}{|c|c|c|c|}
\hline Run & $\alpha$ & $\lambda$ & RA $(\%)$ \\
\hline$\# 1$ & 10 & 0.05 & 99.47 \\
\hline$\# 2$ & 20 & 0.05 & 99.37 \\
\hline$\# 3$ & 30 & 0.05 & 99.55 \\
\hline$\# 4$ & 40 & 0.05 & 99.46 \\
\hline$\# 5$ & 50 & 0.05 & 99.50 \\
\hline$\# 6$ & 10 & 0.1 & 99.37 \\
\hline$\# 7$ & 20 & 0.1 & 99.34 \\
\hline$\# 8$ & 30 & 0.1 & 99.43 \\
\hline$\# 9$ & 40 & 0.1 & 99.58 \\
\hline$\# 10$ & 50 & 0.1 & 99.49 \\
\hline
\end{tabular}

\section{E. Comparing performances of the classification techniques}

The performance of the proposed classifier has been compared with other classifiers for investigating the capability of the proposed classifier, as indicated in Table 8. In this respect, probabilistic neural networks (PNN) [36] and Multilayered perceptron (MLP) neural network with different training algorithm such as: Back propagation (BP) learning algorithm [37] and with Resilient propagation (RP) learning algorithm [38] are considered. They comprise parameters which should be readjusted in any new classification. Furthermore, those parameters regulate the classifiers to be best fitted in for classification task. In most cases, there is no classical method for obtaining the values of them and therefore, they are experimentally specified through try and error. It can be seen from Table 8 that the proposed method has better recognition accuracy than other classifiers.

Table 8. Comparison the performance of proposed method with other classifiers.

\begin{tabular}{|c|c|}
\hline Classifier & Recognition accuracy (\%) \\
\hline PNN & 96.12 \\
\hline MLP (BP) & 95.78 \\
\hline MLP (RP) & 96.28 \\
\hline COA-ANFIS & 99.58 \\
\hline
\end{tabular}

\section{F. Comparison and discussion}

For comparison purposes, Table 9 gives the classification accuracies of our method and previous methods applied to the same database. As can be seen from the results, proposed method obtains a excellent classification accuracy.

Table 9. A summary of different classification algorithms together with their reported results used measures of the accuracy

\begin{tabular}{|c|c|c|c|}
\hline Ref. no & Year & Classifier & RA(\%) \\
\hline$[39]$ & 1992 & MLP & 94.30 \\
\hline$[40]$ & 1994 & LVQ & 97.70 \\
\hline$[10]$ & 1997 & MLP & 99.00 \\
\hline$[41]$ & 1999 & MLP(SPA) & 96.38 \\
\hline$[42]$ & 2000 & MLP & 93.73 \\
\hline$[14]$ & 2003 & MLP & 97.18 \\
\hline$[43]$ & 2006 & MLP & 97.20 \\
\hline$[44]$ & 2008 & MLP(RSFM) & 97.46 \\
\hline$[45]$ & 2008 & PNN & 95.58 \\
\hline$[46]$ & 2009 & MLP & 97.22 \\
\hline This & - & COA-ANFIS & 99.58 \\
work & & & \\
\hline
\end{tabular}

\section{CONCLUSION}

Control chart patterns (CCPs) are important statistical process control tools for determining whether a process is run in its intended mode or in the presence of unnatural patterns. Unnatural CCPs provide clues to potential quality problems at an early stage, to eliminate defects before they are produced. In this paper presents a novel 
hybrid intelligent method for recognition of common types of CCP. The proposed method includes three main modules: the feature extraction module, the classifier module and optimization module. In the feature extraction module, a proper set of eight shape and statistical features is presented that is useful for recognition of control chart patterns. Extraction of these features does not call for utilizing the experience and skill of the users and thus the CCP recognizer developed based of these features will be truly automated. Further, the use of this features set required less training effort and resulted in better recall performance. These confirm the expectation that a feature-based input vector representation results in better recognizer performance. In the classifier module ANFIS techniques was proposed for the comprehensive recognition of CCPs. The structure of the proposed classifier is composed of the two major decision layers. In first layer sextuple patterns of control chart are divided to three groups of double patterns. These groups are: normal and cyclic patterns (Group1), upward shift and upward trend patterns (Group2), downward shift and downward trend patterns (Group3). In the second layer the recognition process is done by shape features and classifiers in each group. For the optimization module, cuckoo optimization algorithm is proposed to improve the generalization performance of the recognizer. In this module, it the ANFIS classifier design is optimized by searching for the best vector of radius. The results showed that the proposed model was effective in finding the parameters of ANFIS, and that it improved classification accuracy. We evaluated the proposed model using a data set and compared it with other models. The simulation results indicate that the proposed method can correctly achieve high classification accuracy $(99.58 \%)$.

\section{REFERENCES}

[1] D.C. Montgomery. Introduction to Statistical Quality Control 2005. 5thed, John Wiley, Hoboken, NJ, USA.

[2] J.A. Swift,J.H. Mize. Out-of-control pattern recognition and analysis for quality control charts using lisp-based systems. Computers and Industrial Engineering 1995; 28: 81-91.

[3] J.R. Evans, W.M. Lindsay. A framework for expert system development in statistical quality control. Computers and Industrial Engineering 1998; 14: 335-343.

[4] T.T. El-Midany. A proposed framework for control chart pattern recognition in multivariate process using artificial neural networks. Expert Systems with Applications 37 (2010) 1035-1042.

[5] S.M.T. Fatemi Ghomi.Recognition of unnatural patterns in process control charts through combining two types of neural networks. Applied Soft Computing 11 (2011) 54445456.

[6] Z. Cheng. Y. Ma.A Research about Pattern Recognition of Control Chart Using Probability Neural Network. 2008 ISECS International Colloquium on Computing, Communication, Control, and Management.

[7] R. S. Guh, Y. R. Shiue, Online identification of control chart patterns using self-organized approaches, International Journal of Production Research 2005;43: $1225-1254$.
[8] C.H. Wang, W. Kuo, H. Qi. An integrated approach for process monitoring using wavelet analysis and competitive neural network. International Journal of Production Research 2007; 45: 227-244.

[9] K. Assaleh. Features extraction and analysis for classifying causable patterns in control charts. Computers \& Industrial Engineering 49 (2005) 168-181.

[10] D.T. Pham, M.A. Wani, Feature-based control pattern recognition, International Journal of Production Research 1997; 35: 1875--1890.

[11] S.K. Gauri, S. Chakraborty. Improved recognition of control chart patterns using artificial neural networks, Int J Adv Manuf Technol 2008; 36: 1191- 1201.

[12] Z. Chen, S. Lu, S. Lam. A hybrid system for SPC concurrent pattern recognition, Advanced engeering informatics 2007; 21: 303-310.

[13] A. Hassan, M.S. Nabi Baksh, A.M. Shaharoun, H. Jamaluddin. Improved SPC chart pattern recognition using statistical features, International Journal of Production Research 2003; 41: 1587-1603.

[14] S. Narayanamoorthy, S.Saranya, S.Maheswari. A Method for Solving Fuzzy Transportation Problem (FTP) using Fuzzy Russell's Method. International Journal of Intelligent Systems and Applications (IJISA). PP.71-75, Pub. Date: 2013-1-3.

[15] Sakshi Bangia, P R Sharma, Maneesha Garg. Simulation of Fuzzy Logic Based Shunt Hybrid Active Filter for Power Quality Improvement. International Journal of Intelligent Systems and Applications (IJISA). PP.96-104, Pub. Date: 2013-1-3.

[16] P.K Bhatia, Surender Singh. A New Measure of Fuzzy Directed Divergence and Its Application in Image Segmentation. International Journal of Intelligent Systems and Applications (IJISA). PP.81-89, Pub. Date: 2013-3-1.

[17] E. Avci, D. Hanbay, A. Varol. An expert Discrete Wavelet Adaptive Network Based Fuzzy Inference System for digital modulation recognition. Expert Systems with Applications 2007; 33: 582-589.

[18] M. Hosoz, H.M. Ertunc, H. Bulgurcu. An adaptive neurofuzzy inference system model for predicting the performance of a refrigeration system with a cooling tower. Expert Systems with Applications 2011; 38: 14148-14155.

[19] M. Sugeno, G.T. Kang, Structure identification of fuzzy model, Fuzzy Sets Syst. 1988; 28: 15-33.

[20] T. Takagi, M. Sugeno, Fuzzy identification of systems and its applications to modeling and control, IEEE Trns. Syst., Man Cybern 1985; 15: 116-132.

[21] T. Takagi, M. Sugeno, Derivation of fuzzy control rules from humanoperator's control actions, in: Proc. IFAC Symp. Fuzzy Inform.,Knowledge Representation and Decision Analysis, July 1985; 55-60.

[22] E.H. Mamdani, S. Assilian, An experiment in linguistic synthesis with afuzzy logic controller, Int. J. Man-Mach. Stud. 1975; $7: 1-13$.

[23] J.S.R. Jang, ANFIS: Adaptive-Network-based Fuzzy Inference Systems, IEEE Trans. Syst., Man Cybern. 23 (May/June (3)) .1993; 665-685.

[24] S. Haykin, Neural Networks-A Comprehensive Foundation, second ed.,Prentice-Hall of India Pvt. Ltd., New Delhi, India, 2003.

[25] J.M. Zurada, Introduction to Artificial Neural Systems, PWS PublicationCompany, 1992.

[26] M.T. Hagan, H.B. Demuth, M.H. Beale, Neural Network Design, PWSPublishing, Boston, MA, 1996.

[27] S. Chiu, Fuzzy model identification based on cluster estimation, J. Intell.Fuzzy Syst. 1994; 2 (3): 267-278. 
[28] S. Chiu, Selecting input variables for fuzzy models, J. Intell. Fuzzy Syst. 1996; 4(4): 243-256.

[29] M. Buragohain , C. Mahanta. A novel approach for ANFIS modelling based on full factorial design. Applied Soft Computing 2008; 8: 609-625.

[30] R. Rajabioun. Cuckoo Optimization Algorithm. Applied Soft Computing 2011; 11: 5508-5518.

[31] http://archive.ics.uci.edu/ml/databases/synthetic control/synthetic control. data.html.

[32] D. Karaboga, B. Basturk, On the performance of artificial bee colony $(\mathrm{ABC})$ algorithm, Applied Soft Computing 2008; 8: 687-697.

[33] K.S. Tang, K.F. Man, S. Kwong, Q. He, Genetic algorithms and their applications, IEEE Signal Processing Magazine 1996; 13: 22-37.

[34] J. Kennedy, R. Eberhart, Particle swarm optimization, in: Proceedings of IEEE International Conference on Neural Networks 1995; 4:1942-1948.

[35] R. Rajabioun, F. Hashemzadeh, E. Atashpaz-Gargari. Colonial competitive algorithm A novel approach for PID controller design in MIMO distillation column process. International Journal of Intelligent Computing and Cybernetics 2008; 3: 337-355.

[36] D.F. Specht, Probabilistic neural networks, Neural Networks 1990; 109-118.

[37] S.Haykin, Neural Networks: A Comprehensive Foundation, Mac Millan, New York, 1999.

[38] M. Riedmiller, H. Braun, A direct adaptive method for faster back propagation learning: the RPROP algorithm, in: Proceedings of the IEEE Int. Conf. On Neural Networks, SanFrancisco, CA, March28, 1993.

[39] D. T. Pham, E. Oztemel, Control chart pattern recognition using neural networks, Journal of Systems Engineering 1992; 2: 256-262.

[40] D. T. Pham, E. Oztemel, Control chart pattern recognition using linear vector quantization networks, International Journal of Production Research 1994; 256-262.

[41] R.S. Guh, J.D.T. Tannock. A neural network approach to characterize pattern parameters in process control.

[42] S. Sagiroujlu, E. Besdoc, M. Erler, Contro chart pattern recognition using artificial neural networks, Turkish Journal of Electrical Engineering 2000; 8: 137-147.

[43] S. Gauri a, S. Chakraborty. Feature-based recognition of control chart patterns. Computers \& Industrial Engineering 2006; 51: 726-742.

[44] Q. Le, X. Goal, L. Teng, M. Zhu, A new ANN model and its application in pattern recognition of control charts, in: Proc. IEEE. WCICA, 2008; 1807-1811.

[45] Z. Cheng, Y. Ma, A research about pattern recognition of control chart using probability neural network, in: Proc. ISECS, 2008; 140-145.

[46] S. Gauri a, S. Chakraborty. Recognition of control chart patterns using improved selection of features. Computers \& Industrial Engineering 2009; 56: 1577-1588.

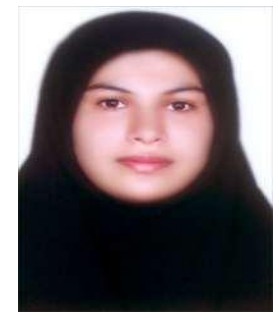

Somayeh Mirzaei was born in 1989 , Iran. $\mathrm{He}$ is student of electrical engineering in Shams University, Gonbad Kavous, Iran. Her research interests include Soft computing, roboric and web. ID: somayeh.mirzaei.shams@gmail.com

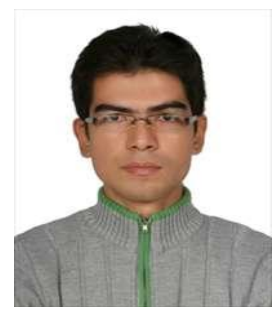

Massoud Pourmandi was born in 1989, Iran. He received BS degree in electronic engineering from the University of Guilan and M.Sc. student of Electrical Engineering- Communication Systems in Ferdowsi University of Mashhad. ID: massoud.pourmandi@gmail.com

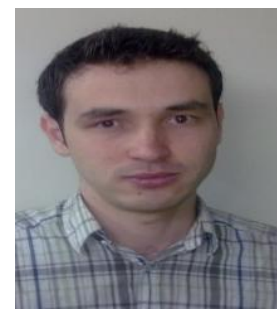

Jalil Addeh was born in 1986, Iran. He received B.S. degree in electrical engineering from Babol University of Technology, Iran, in 2010, and the M.S. degree in electrical engineering (control) from Babol University of Technology, Babol, Iran, in 2012. His research interests include pattern recognition, Soft computing, fuzzy logic, neural networks and nonlinear control.

ID: jalil_addeh@yahoo.com

\section{Authors' Profiles}

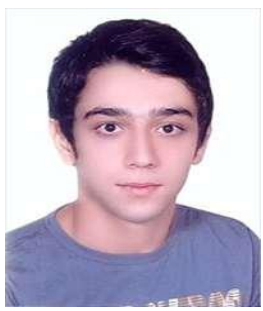

Abdolhakim Nikpey was born in 1994, Iran. He is student of electrical engineering in Shams University, Gonbad Kavous, Iran. His research interests include Soft computing, roboric and web .

ID: nikpey72@gmail.com 